\title{
Plant-borne compounds and nanoparticles: challenges for medicine, parasitology and entomology
}

\author{
Giovanni Benelli ${ }^{1,2}$
}

Received: 9 August 2017 / Accepted: 11 August 2017 / Published online: 30 March 2018

(C) Springer-Verlag GmbH Germany 2017

The effective and eco-friendly control of pathogens and parasites is a major challenge for parasitologists and entomologists worldwide. The fast-growing development of resistance to drugs and pesticides currently used highlighted the urgent need of developing newer and safer control tools. Herbal preparations represent a huge source of bioactive principles showing high efficacy against many pathogens, parasites and pests, as elucidated by the recent Nobel Prize to the Chinese scientist Y. Tu for the discovery of the antimalarial drug artemisinin. Therefore, the extensive screening of the bioactivity of herbal preparations is often helpful to develop botanical-based pesticides and drugs.

Furthermore, nanosized materials possess stunning physical and chemical properties, with special reference to their optical and catalytic ones. Therefore, they play a promising role in the development of novel and effective drugs and pesticides. However, the synthesis of nanosized materials is traditionally carried out with chemical and physical methods that need the employ of highly toxic chemicals or high-energy inputs. To lower the environmental impact of these processes, many researchers recently focused on the so-called green nanosynthesis, where microbial, plant-borne or fungal compounds can

Responsible editor: Philippe Garrigues

Giovanni Benelli

benelli.giovanni@gmail.com

1 Department of Agriculture, Food and Environment, University of Pisa, Via del Borghetto 80, 56124 Pisa, Italy

2 The BioRobotics Institute, Scuola Superiore Sant'Anna, Viale Rinaldo Piaggio 34, 56025 Pontedera, Pisa, Italy be used as reducing and capping agents during the fabrication of nanoparticles with multipurpose effectiveness. These synthesis routes are cheap, one-pot and often lead to the production of nanomaterials with controlled size and shape, two features that are extremely important routing the nanoparticles' bioactivity.

Despite the promising potential of plant-borne pesticides and drugs, as well as green-synthesized nanomaterials, their applications in the "real world" are still limited and further research is urgently needed. In this framework, Environmental Science and Pollution Research (ESPR) has featured a collection of innovative papers highlighting emerging environmental trends in "Plant-borne compounds and nanoparticles: challenges for medicine, parasitology and entomology" (GREEN-NANOPEST\&DRUGS). This Special Issue included both original research and review articles on the prevention and control of arthropod pests and arthropod-borne diseases with ecofriendly materials, including nanoscale ones. The effective management of microbial pathogens is also covered.

Major emphasis has been devoted to the impact of botanical pesticides on non-target organisms and the environment, their sub-lethal effects, including genotoxicity and behavioural modifications, the toxicity of green-synthesized nanopesticides on non-target organisms and the environment, 
the utilization of industrial byproducts as sources of pesticides or repellents against arthropod vectors and microbial pathogens, and their side effects.

The Special Issue GREEN-NANO-PEST\&DRUGS, in addition to the specific issues related to the development of green pesticides and drugs, specifically addressed the risks and challenges connected with the employ of these materials in the "real world", which need input from ecotoxicologists, entomologists, parasitologists and researchers working in nanomaterial science.

I am aware that this Special Issue cannot fully reflect the diversity and creativity of the ideas and new insights rapidly growing in the field of green nanosynthesis research. On the other hand, as Editor, I hope that GREEN-NANOPEST\&DRUGS may boost further research on the impact of green nanoscience in the fields of parasitology and entomology, with special reference to the standardization of nanosynthesis routes, the formulation of green pesticides and drugs with long-lasting efficacy and clear mechanisms of action, understanding the fate of nanoparticles in the field as well as in aquatic environments, and the ecotoxicological issues related to their non-target effects at sub-lethal doses.

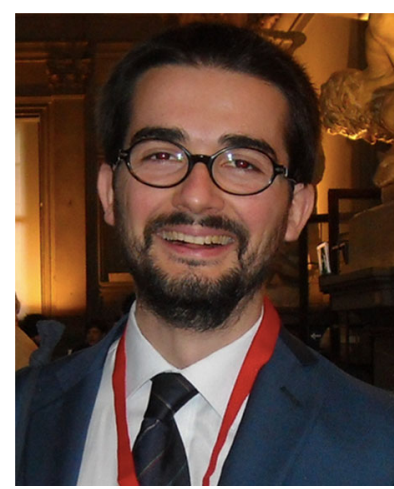

Dr. Giovanni Benelli got an International Ph.D. in Agrarian and Veterinary Sciences at the University of Pisa and Sant'Anna School of Advanced Studies, Pisa. He has worked in several international institutions including the University of Hawaii at Manoa (USA) and the University of Jaén (Spain). Dr. Benelli serves as a research entomologist and lecturer at the University of Pisa. He has been also appointed as affiliated researcher at The BioRobotics Institute, Sant'Anna School of Advanced Studies. His research focuses on general and applied entomology, ecotoxicology and biological control, with a major focus on the ecofriendly control of arthropod vectors of medical and veterinary importance. Giovanni serves as academic editor and editorial board member for many international journals. He has published more than 300 papers in international journals with impact factor. Currently, he cooperates with more than 80 researchers worldwide on various research projects, including FP7 Collective Cognitive Robots, H2020 subCULTron and Taiwan National Nano Project, MOST-105-2119-M-008-006. Dr. Benelli received several prizes from national and international organizations, including the International Organisation for Biological Control, Accademia dei Georgofili and the Italian National Academy of Entomology. 